\title{
Molecular Surveillance of Cronobacter spp. Isolated from a Wide Variety of Foods from 44 Different Countries by Sequence Typing of 16S rRNA, rpoB and O-Antigen Genes
}

\author{
Nancy Miranda ${ }^{1}$, Pratik Banerjee ${ }^{2}$, Steven Simpson ${ }^{1}$, Khalil Kerdahi ${ }^{1}$ \\ and Irshad M. Sulaiman 1,* \\ 1 Southeast Regional Laboratory, U.S. Food and Drug Administration, Atlanta, GA 30309, USA; \\ Nancy.Vega@fda.hhs.gov (N.M.); Steven.Simpson@fda.hhs.gov (S.S.); Khalil.Kerdahi@fda.hhs.gov (K.K.) \\ 2 Division of Epidemiology, Biostatistics, and Environmental Health, School of Public Health, \\ University of Memphis, Memphis, TN 38152, USA; pbnerjee@memphis.edu \\ * Correspondence: Irshad.Sulaiman@fda.hhs.gov; Tel.: +1-404-575-1523
}

Academic Editor: Debabrata Biswas

Received: 4 April 2017; Accepted: 8 May 2017; Published: 11 May 2017

\begin{abstract}
Cronobacter spp. are emerging infectious bacteria that can cause acute meningitis and necrotizing enterocolitis in neonatal and immunocompromised individuals. Although this opportunistic human-pathogenic microorganism has been isolated from a wide variety of food and environmental samples, it has been primarily linked to foodborne outbreaks associated with powdered infant formula. The U.S. Food and Drug Administration use the presence of these microbes as one of the criteria to assess food adulteration and to implement regulatory actions. In this study, we have examined 195 aliquots of enrichments from the nine major categories of foods (including baby and medical food, dairy products, dried food, frozen food, pet food, produce, ready-to-eat snacks, seafood, and spices) from 44 countries using conventional microbiological and molecular techniques. The typical colonies of Cronobacter were then identified by VITEK2 and real-time PCR. Subsequently, sequence typing was performed on the 51 recovered Cronobacter isolates at the $16 \mathrm{~S}$ rRNA, rpoB and seven O-antigen loci for species identification in order to accomplish an effective surveillance program for the control and prevention of foodborne illnesses.
\end{abstract}

Keywords: Cronobacter spp.; isolation; chromogenic and traditional media; molecular typing; foodborne disease

\section{Introduction}

Cronobacter spp. is a group of Gram-negative bacteria belonging to the family Enterobacteriacea which can survive in environments with extremely dry conditions. It is considered an emerging opportunistic pathogen capable of causing severe infections including necrotizing enterocolitis, bacteremia, and meningitis in humans [1-3]. In addition, this multi-species complex is typically facultative anaerobic, oxidase-negative, catalase-positive, rod-shaped, motile, non-spore forming bacteria that can predominantly produce a yellow pigment. Even though seven species of Cronobacter have been described (including C. sakazakii, C. muytjensii, C. turicensis, C. dublinensis, C. malonaticus, C. universalis, and C. condimenti), only three of these species (C. turicensis, C. malonaticus, and C. Sakazakii) have been associated with cases of infant death [4-7]. Importantly, a very wide temperature range $\left(6\right.$ to $\left.-45^{\circ} \mathrm{C}\right)$ has been reported for the typical growth of this group in brain heart infusion broth [4]. The growth of this group of organisms has also been recorded in powder infant formula (PIF) 
reconstituted at temperatures ranging from 8 to $47^{\circ} \mathrm{C}$ [8]. It can survive for longer than two years in a desiccated state [3].

Although the natural reservoir of this organism is still unsettled, it has been isolated from a variety of food matrices that included produce, spices, herbs, and animal feed [9]. Nevertheless, the PIF has been associated with mostly neonate cases $[10,11]$. Furthermore, with the exception of $C$. condimenti, the rest of the six Cronobacter species have been associated with clinical infections; the C. sakazakii and C. malonaticus isolates have been reported to be primarily responsible for causing the majority of infant illnesses [6,7,12].

Thus far, DNA sequencing is considered the gold standard for rapid detection and species identification of human-pathogenic microorganisms of public health importance [13-15]. It has also been used to understand the population genetic structure, phylogenetic relationship, and taxonomic revision of various human-pathogenic bacteria causing foodborne illness. More recently, nucleotide sequence characterization is routinely employed in effective epidemiologic studies to reveal transmission routes of emerging infectious diseases, and in the prevention and control of various foodborne and waterborne diseases [13-16]. Multilocus sequence typing has been successfully used in the species identification of Cronobacter spp. worldwide [17].

Recently, we analyzed 195 food samples belonging to nine major food categories (including baby and medical food, dairy products, dried food, frozen food, pet food, produce, seafood, spices and ready-to-eat snacks), originated from 44 countries located on five continents (Americas, Africa, Asia, Europe, and Oceania) for the presence of Salmonella, Listeria monocytogenes, and E. coli in foods as part of an ongoing surveillance program for food safety of the agency (unpublished). In this study, we have tested the 195 aliquots of the above food enrichments for the presence of Cronobacter, and we have performed a two-step enrichment to aid the injured cells. Afterwards, the secondary enrichments were streaked on three chromogenic media and incubated at different temperatures to reduce the background flora and increase the odds of recovering the organism. Molecular typing was conducted on the recovered Cronobacter isolates for species identification by DNA sequencing of $16 \mathrm{~S}$ rRNA, rpoB and seven sets of $\mathrm{O}$-antigen loci.

\section{Materials and Methods}

\subsection{Food Samples}

This study examined a total of 195 aliquots of enrichments for the presence of Cronobacter. As listed in Table 1, the examined foods included baby and medical food (6 samples from 2 countries), dairy products (11 samples from 2 countries), dried food ( 7 samples from 5 countries), frozen food ( 5 samples from 3 countries), pet food ( 24 samples from 6 countries), produce (56 samples from 14 countries), seafood (11 samples from 9 countries), spices ( 54 samples from 21 countries), and ready-to-eat snacks (21 samples from 12 countries). The enrichments were initially made to isolate Salmonella, Listeria monocytogenes, and E. coli from the food samples in order to conduct a routine surveillance program for food safety of the agency, following the FDA Bacteriological Analytical Manual (BAM) [18].

Table 1. Food samples analyzed in the study with their countries of origin.

\begin{tabular}{cl}
\hline Origin (Region/Country) & \multicolumn{1}{c}{ Food Products Tested } \\
\hline Americas & chia seed, pet food \\
Argentina & pet food, papaya \\
Belize & papaya \\
Brazil & pet food, sesame seed \\
Canada & chili powder \\
Chile & basil \\
Colombia & papaya, coriander \\
Costa Rica & cantaloupe, cilantro, cucumber, papaya \\
\hline
\end{tabular}


Table 1. Cont.

\begin{tabular}{|c|c|}
\hline Origin (Region/Country) & Food Products Tested \\
\hline \multicolumn{2}{|l|}{ Americas } \\
\hline Ecuador & ready-to-eat snack \\
\hline El Salvador & okra, spice powder \\
\hline Guatemala & breading flour, mango, papaya, seasoned flour \\
\hline Guyana & brown sauce \\
\hline Haiti & mango \\
\hline Honduras & cucumber \\
\hline Jamaica & spice powder \\
\hline Mexico & avocado, basil, cilantro, kale, octopus, yellow croaker \\
\hline Nicaragua & cheese \\
\hline Peru & paprika powder, shrimp \\
\hline USA & $\begin{array}{l}\text { alfalfa sprout, avocado, broccoli sprout, broccoli sprout seed, cheese, } \\
\text { clover seed, cucumber, frozen ravioli, kale, organic clover sprout, } \\
\text { parsley, pet food, powder infant formula, powder milk, ready-to-eat } \\
\text { snack, spice powder, spinach, tomato }\end{array}$ \\
\hline \multicolumn{2}{|l|}{ Africa } \\
\hline Sao Vicente (Cape Verde) & ready-to-eat snack \\
\hline Ghana & ogbono seed, smoked tilapia, spice \\
\hline Kenya & spice powder \\
\hline Morocco & ready-to-eat snack \\
\hline South Africa & pepper, spice powder \\
\hline \multicolumn{2}{|l|}{ Asia } \\
\hline China & $\begin{array}{l}\text { cauliflower, frozen crab cake, garlic powder, pet food, spice } \\
\text { powder, tilapia }\end{array}$ \\
\hline India & $\begin{array}{l}\text { black pepper, crushed red pepper, garlic powder, sesame seed, spice } \\
\text { powder, ready-to-eat snack, wafers wheels }\end{array}$ \\
\hline Indonesia & spice powder, tilapia \\
\hline Israel & basil \\
\hline Malaysia & shrimp \\
\hline Pakistan & ready-to-eat snack, spice powder \\
\hline Philippines & cassava leaf, desiccated coconut \\
\hline Sri Lanka & cinnamon, cinnamon quill \\
\hline South Korea & ready-to-eat snack \\
\hline Taiwan & black sesame powder, spice salt, spice powder \\
\hline Thailand & ready-to-eat snack, spice powder \\
\hline Turkey & laurel leaves, strawberry \\
\hline Vietnam & ground black pepper, ready-to-eat snack, shrimp, tuna, white pepper \\
\hline \multicolumn{2}{|l|}{ Europe } \\
\hline Germany & chocolate powder, spice powder \\
\hline Ireland & pet food, ready-to-eat snack \\
\hline Italy & frozen linguine, ready-to-eat snack \\
\hline Netherland & parsley leaf \\
\hline Spain & cantaloupe, crawfish, paprika \\
\hline UK & medical food, salmon \\
\hline \multicolumn{2}{|l|}{ Oceania } \\
\hline Australia & alfalfa beans \\
\hline
\end{tabular}

\subsection{Pre-Enrichment, Secondary Enrichment, and Culture on Chromogenic Media}

In order to recover the Cronobacter spp. from the enrichment, aliquots of one $\mathrm{mL}$ of the refrigerated enrichment broth were aseptically added to $9 \mathrm{~mL}$ of pre-warmed BPW (at $43.5^{\circ} \mathrm{C}$ in an incubator), and incubated at $37^{\circ} \mathrm{C}$ for $16-24 \mathrm{~h}$. After incubation, one $\mathrm{mL}$ portions of the enrichment were then transferred to $9 \mathrm{~mL}$ of R\&F Enterobacter sakazakii enrichment broth (R \& F Laboratories, Downers Grove, IL, USA) and $9 \mathrm{~mL}$ of Al-Holy-Rasco (AR) broth, and incubated at $43.5 \pm 0.5^{\circ} \mathrm{C}$ for $24 \pm 2 \mathrm{~h}$, as described above [19]. After completion of incubation, a loop full of R\&F and AR enrichment broths were plated onto the Druggan-Forsythe-Iversen (DFI, Oxoid, Basingstoke, UK) and R\&F 
Enterobacter sakazakii (Cronobacter) chromogenic agar plates by streaking at least three quadrants followed by incubation overnight, at $36 \pm 1{ }^{\circ} \mathrm{C}$ for the DFI agar and $42 \pm 1{ }^{\circ} \mathrm{C}$ for the R\&F E. sakazakii agar respectively. After visualizing typical colonies on DFI and R\&F agar plates, the colonies were transferred to Enterobacter sakazakii isolation agar (ESIA) (Oxoid, UK) and incubated at $43.5 \pm 0.5^{\circ} \mathrm{C}$ for 18-24 $\mathrm{h}$ following manufacturers' recommendation for the reduction of background flora and better isolation of the target organism. The typical colonies isolated from ESIA were then transferred to Trypticase Soy Agar (TSA) with 5\% Sheep Blood for identification and molecular analysis.

\subsection{Isolate Identification}

Identification of recovered isolates was achieved by making a bacterial suspension of purified culture raised on the TSA plates with 5\% Sheep Blood. The colonies were suspended in $3 \mathrm{~mL}$ of sterile $0.45 \%$ saline, and its turbidity was verified and adjusted to achieve the necessary optical density for analysis using Biomérieux Vitek 2 System with the Vitek GN cards that range from 0.50-0.63 McFarland, following manufacturer's instructions [20]. Once the run was completed, the isolates identified as the "Cronobacter sakazakii group" were transferred to BHI broth and incubated overnight at $37 \pm 1{ }^{\circ} \mathrm{C}$, for DNA extraction.

\subsection{DNA Extraction and Real-Time PCR}

The DNA extraction was achieved by using QIAGEN DNeasy Blood and Tissue kit following manufacturer's protocol for the purification of total DNA from Gram-negative bacteria (QIAGEN, Valencia, CA, USA) for all of the food samples that tested positive for Cronobacter in the study (Table 2). For each isolate, the cell pellet from one milliliter bacterial culture grown overnight at $37^{\circ} \mathrm{C}$ in BHI broth was used as the starting material. The concentration of purified genomic DNA was measured at $260 \mathrm{~nm}$ absorbance using a NanoDrop-1000 spectrophotometer (NanoDrop Technology, Rockland, DE, USA), and stored at $-20{ }^{\circ} \mathrm{C}$ until used.

To perform the real time PCR analysis, two microliters of sample templates were examined following the FDA BAM Method without internal and using the Cepheid SmartCycler Thermal Cycler (software version $2.0 \mathrm{~d}$, Cepheid, Sunnyvale, CA, USA) [18]. 
Table 2. Food samples that tested positive for Cronobacter in the study.

\begin{tabular}{|c|c|c|c|c|c|c|c|c|c|c|c|}
\hline \multirow{2}{*}{ S. No. } & \multirow{2}{*}{$\begin{array}{l}\text { Sample } \\
\text { Number }\end{array}$} & \multirow{2}{*}{$\begin{array}{l}\text { Description of Food } \\
\text { Products }\end{array}$} & \multirow{2}{*}{$\begin{array}{c}\text { Country of } \\
\text { Origin }\end{array}$} & \multirow{2}{*}{$\begin{array}{l}\text { Food Product } \\
\text { Type/Category }\end{array}$} & \multirow{2}{*}{$\begin{array}{l}\text { Bacterial } \\
\text { Culture }\end{array}$} & \multirow{2}{*}{ QPCR } & \multicolumn{3}{|c|}{ PCR Screening } & \multirow{2}{*}{$\begin{array}{c}{ }^{*} \text { O-antigen Reference } \\
\text { (Sequence Variation, \% Similarity) }\end{array}$} & \multirow{2}{*}{$\begin{array}{l}\text { *O-antigen } \\
\text { Sequence Typ }\end{array}$} \\
\hline & & & & & & & 16S rRNA & rpoB & ${ }^{*} \mathrm{O}$-antigen & & \\
\hline 1 & SRL-66 & Cassava leaf & Philippines & Dried food & TG & + & + & + & 6 & JQ674749, This report (Identical) & C. sakazakii \\
\hline 2 & SRL-80 & Breading flour & Guatemala & Dried food & TG & + & + & + & 1 & CP011047, This report (Identical) & C. sakazakii \\
\hline 3 & SRL-154 & Frozen Ravioli & USA & Frozen food & TG & + & + & + & 6 & JQ674749, This report (4-point-mutation) & C. sakazakii \\
\hline 4 & SRL-86 & Pet food & USA & Pet food & TG & + & + & + & 2 & EU076546, This report (Identical) & C. sakazakii \\
\hline 5 & SRL-91 & Pet food & Canada & Pet food & TG & + & + & + & NOA & NOA & NOA \\
\hline 6 & SRL-93 & Pet food & Canada & Pet food & TG & + & + & + & 1 & CP000783, This report (Identical) & C. sakazakii \\
\hline 7 & SRL-101 & Pet food & Canada & Pet food & TG & + & + & + & 1 & CP000783, This report (Identical) & C. sakazakii \\
\hline 8 & SRL-163 & Pet food & China & Pet food & TG & + & + & + & 2 & EU076546, This report (Identical) & C. sakazakii \\
\hline 9 & SRL-186 & Pet food & USA & Pet food & TG & + & + & + & NOA & NOA & NOA \\
\hline 10 & SRL-199 & Pet food & China & Pet food & TG & + & + & + & 1 & CP011047, This report (Identical) & C. sakazakii \\
\hline 11 & SRL-12 & Basil & Mexico & Produce & TG & + & + & + & NOA & NOA & NOA \\
\hline 12 & SRL-13 & Parsley & USA & Produce & TG & + & + & + & NOA & NOA & NOA \\
\hline 13 & SRL-20 & Basil & Colombia & Produce & TG & + & + & + & 1 & СР000783, This report (1-point-mutation) & C. sakazakii \\
\hline 14 & SRL-35 & Alfalfa beans & Australia & Produce & TG & + & + & + & 1 & CP011047, This report (Identical) & C. sakazakii \\
\hline 15 & SRL-42 & Basil & Colombia & Produce & TG & + & + & + & NOA & NOA & NOA \\
\hline 16 & SRL-87 & Parsley leaf & Netherland & Produce & TG & + & + & + & 2 & EU076546, This report (Identical) & C. sakazakii \\
\hline 17 & SRL-94 & Alfalfa sprout & USA & Produce & TG & + & + & + & NOA & NOA & NOA \\
\hline 18 & SRL-140 & Avocado & USA & Produce & TG & + & + & + & 6 & JQ674749, This report (Identical) & C. sakazakii \\
\hline 19 & SRL-173 & Avocado & USA & Produce & TG & + & + & + & 3 & HQ646169, This report (Identical) & C. sakazakii \\
\hline 20 & SRL-194 & Avocado & USA & Produce & TG & + & + & + & 6 & JQ674749, This report (4-point-mutation) & C. sakazakii \\
\hline 21 & SRL-95 & Smoked Tilapia & Ghana & Seafood & TG & + & + & + & 7 & JQ674750, This report (2-point-mutation) & C. sakazakii \\
\hline 22 & SRL-4 & Garlic powder & India & Spice & TG & + & + & + & 2 & EU076546, This report (Identical) & C. sakazakii \\
\hline 23 & SRL-36 & Spice powder & Pakistan & Spice & TG & + & + & + & 3 & HQ646169, This report (Identical) & C. sakazakii \\
\hline 24 & SRL-40 & Spice powder & South Africa & Spice & TG & + & + & + & 2 & EU076546, This report (Identical) & C. sakazakii \\
\hline 25 & SRL-41 & Pepper & South Africa & Spice & TG & + & + & + & 1 & CP011047, This report (Identical) & C. sakazakii \\
\hline 26 & SRL-43 & Spice salt & Taiwan & Spice & TG & + & + & + & 2 & EU076546, This report (Identical) & C. sakazakii \\
\hline 27 & SRL-47 & Black sesame powder & Taiwan & Spice & TG & + & + & + & 2 & EU076546, This report (Identical) & C. sakazakii \\
\hline 28 & SRL-56 & Spice powder & India & Spice & TG & + & + & + & 1 & CP011047, This report (Identical) & C. sakazakii \\
\hline 29 & SRL-65 & Spice powder & India & Spice & TG & + & + & + & 4 & JQ674747, This report (Identical) & C. sakazakii \\
\hline 30 & SRL-77 & Spice powder & India & Spice & TG & + & + & + & 1 & CP011047, This report (Identical) & C. sakazakii \\
\hline 31 & SRL-81 & Spice powder & Pakistan & Spice & TG & + & + & + & 7 & JQ674750, This report (2-point-mutation) & C. sakazakii \\
\hline 32 & SRL-82 & Spice powder & India & Spice & TG & + & + & + & 3 & HQ646169, This report (Identical) & C. sakazakii \\
\hline 33 & SRL-102 & Ogbono seed & Ghana & Spice & TG & + & + & + & NOA & NOA & NOA \\
\hline 34 & SRL-104 & Spice powder & Jamaica & Spice & TG & + & + & + & 1 & CP011047, This report (Identical) & C. sakazakii \\
\hline 35 & SRL-109 & Paprika powder & Peru & Spice & TG & + & + & + & 2 & EU076546, This report (Identical) & C. sakazakii \\
\hline 36 & SRL-124 & Spice powder & Germany & Spice & TG & + & + & + & 2 & EU076546, This report (Identical) & C. sakazakii \\
\hline 37 & SRL-126 & Chili powder & Chile & Spice & TG & + & + & + & NOA & NOA & NOA \\
\hline 38 & SRL-128 & Spice powder & Kenya & Spice & TG & + & + & + & 6 & JQ674749, This report (identical) & C. sakazakii \\
\hline 39 & SRL-160 & Spice powder & El Salvador & Spice & TG & + & + & + & 3 & HQ646169, This report (Identical) & C. sakazakii \\
\hline 40 & SRL-171 & Spice powder & India & Spice & TG & + & + & + & 1 & CP011047, This report (Identical) & C. sakazakii \\
\hline 41 & SRL-172 & Spice powder & India & Spice & TG & + & + & + & 6 & JQ674749, This report (Identical) & C. sakazakii \\
\hline 42 & SRL-179 & Spice powder & India & Spice & TG & + & + & + & $\mathrm{NOA}$ & NOA & NOA \\
\hline
\end{tabular}


Table 2. Cont

\begin{tabular}{|c|c|c|c|c|c|c|c|c|c|c|c|}
\hline \multirow{2}{*}{ S. No. } & \multirow{2}{*}{$\begin{array}{l}\text { Sample } \\
\text { Number }\end{array}$} & \multirow{2}{*}{$\begin{array}{l}\text { Description of Food } \\
\text { Products }\end{array}$} & \multirow{2}{*}{$\begin{array}{l}\text { Country of } \\
\text { Origin }\end{array}$} & \multirow{2}{*}{$\begin{array}{l}\text { Food Product } \\
\text { Type/Category }\end{array}$} & \multirow{2}{*}{$\begin{array}{l}\text { Bacterial } \\
\text { Culture }\end{array}$} & \multirow{2}{*}{ QPCR } & \multicolumn{3}{|c|}{ PCR Screening } & \multirow{2}{*}{$\begin{array}{c}* \text { O-antigen Reference } \\
\text { (Sequence Variation, \% Similarity) }\end{array}$} & \multirow{2}{*}{$\begin{array}{c}\text { *O-antigen } \\
\text { Sequence Type }\end{array}$} \\
\hline & & & & & & & 16S rRNA & rpo B & ${ }^{*} \mathrm{O}$-antigen & & \\
\hline 43 & SRL-180 & Spice powder & India & Spice & TG & + & + & + & 6 & JQ674749, This report (Identical) & C. sakazakii \\
\hline 44 & SRL-181 & Spice powder & India & Spice & TG & + & + & + & 2 & EU076546, This report (Identical) & C. sakazakii \\
\hline 45 & SRL-187 & Spice powder & India & Spice & TG & + & + & + & 2 & EU076546, This report (Identical) & C. sakazakii \\
\hline 46 & SRL-51 & Ready-to-eat snack & India & Snack & TG & + & + & + & 4 & JQ674747, This report (Identical) & C. sakazakii \\
\hline 47 & SRL-72 & Ready-to-eat snack & Vietnam & Snack & TG & + & + & + & 2 & EU076546, This report (Identical) & C. sakazakii \\
\hline 48 & SRL-79 & Ready-to-eat snack & India & Snack & TG & + & + & + & 2 & EU076546, This report (Identical) & C. sakazakii \\
\hline 49 & SRL-99 & Ready-to-eat snack & USA & Snack & TG & + & + & + & 1 & CP000783, This report (Identical) & C. sakazakii \\
\hline 50 & SRL-131 & Ready-to-eat snack & India & Snack & TG & + & + & + & 6 & JQ674749, This report (Identical) & C. sakazakii \\
\hline 51 & SRL-152 & Ready-to-eat snack & India & Snack & TG & + & + & + & 1 & СР011047, This report (Identical) & C. sakazakii \\
\hline
\end{tabular}

TG: growth of typical Cronobacter colonies observed on culture plates; * seven sets of primer were used to amplify the O-antigen 1-7 serotypes; NOA: no O-antigen PCR amplification;

+: PCR positive. QPCR: quantitative polymerase chain reaction, also known as real-time polymerase chain reaction (Real-Time PCR). 


\subsection{PCR Amplification}

To amplify the regions of $16 \mathrm{~S}$ rRNA, rpoB and the seven known C. sakazakii-specific O-antigen genes, nine unique PCR protocols were developed using the published PCR primer sets (Table 3). For 16S rRNA PCR amplification, a total of $50 \mu \mathrm{L}$ PCR reaction consisted of $25 \mu \mathrm{L}$ of HotStarTaq Master Mix (QIAGEN, this premixed solution contains HotStarTaq DNA Polymerase, PCR Buffer, and dNTPs with a final concentration of $1.5 \mathrm{mM} \mathrm{MgCl}_{2}$ and $200 \mu \mathrm{M}$ each dNTP), and $25 \mu \mathrm{L}$ of a solution containing $200 \mathrm{nM}$ of each primer, $1.5 \mathrm{mM}$ of additional $\mathrm{MgCl}_{2}$ (Promega, Madison, WI, USA) and template DNA (50 ng) diluted in PCR grade water. The PCR reactions were run for 35 cycles (each cycle is $94{ }^{\circ} \mathrm{C}$ for $45 \mathrm{~s}, 60^{\circ} \mathrm{C}$ for $45 \mathrm{~s}$, and $72{ }^{\circ} \mathrm{C}$ for $60 \mathrm{~s}$ ) in a GeneAmp PCR 9700 thermocycler (Applied Biosystems, Foster City, CA, USA), with an initial hot start $\left(94^{\circ} \mathrm{C}\right.$ for $\left.15 \mathrm{~min}\right)$ and a final extension $\left(72^{\circ} \mathrm{C}\right.$ for $\left.10 \mathrm{~min}\right)$. The PCR conditions for $r p o \mathrm{~B}$ and $\mathrm{O}$-antigen amplification were similar to $16 \mathrm{~S}$ rRNA except that the annealing temperature was $56^{\circ} \mathrm{C}$ for $r p o B$ and it was $50^{\circ} \mathrm{C}$ for the O-antigen PCR amplification. The PCR products were examined by agarose gel electrophoresis and visualized after ethidium bromide staining (Figure 1).

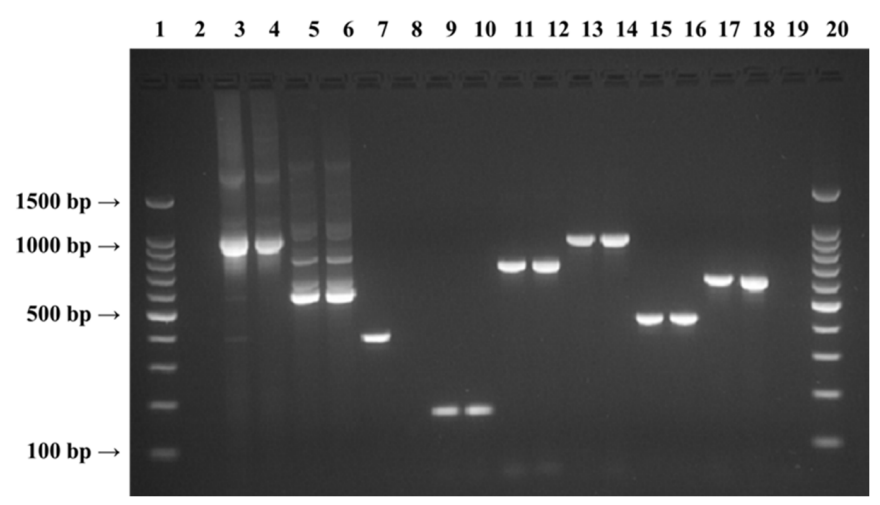

Figure 1. Agarose gel showing Cronobacter-specific PCR amplified products at eight different loci. Lane 1 and 20: Promega ${ }^{\mathrm{TM}} 100$ bp DNA ladder molecular weight marker, Lane 3-4: 16S rRNA, Lane 5-6: rpoB, Lane 7: O-antigen 1, Lane 9-10: O-antigen 2, Lane 11-12: O-antigen 3, Lane 13-14: O-antigen 4, Lane 15-16: O-antigen 6, Lane 17-18: O-antigen 7, and Lane 2-8-19: Negative Control.

Table 3. Published primers used in the study.

\begin{tabular}{|c|c|c|c|}
\hline Target & Primer Name & Primer Sequence $\left(5^{\prime}-3^{\prime}\right)$ & Reference \\
\hline * $16 \mathrm{~S}$ rRNA & $\begin{array}{l}616 \mathrm{~V} \\
630 \mathrm{R}\end{array}$ & $\begin{array}{l}\text { AGAGTTGATYMTGGCTC } \\
\text { CAKAAAGGAGGTGATCC }\end{array}$ & [21] \\
\hline * rpoB & $\begin{array}{l}\text { rpoB-F } \\
\text { rpoB-R }\end{array}$ & $\begin{array}{l}\text { AACCAGTTCCGCGTTGGCCTGG } \\
\text { CCTGAACAACACGCTCGGA }\end{array}$ & [22] \\
\hline ** Wzy, Serotype O1 & $\begin{array}{l}\text { wl-35646 } \\
\text { wl-35647 }\end{array}$ & $\begin{array}{l}\text { CCCGCTTGTATGGATGTT } \\
\text { CTTTGGGAGCGTTAGGTT }\end{array}$ & [23] \\
\hline${ }^{* *} W z y$, Serotype O2 & $\begin{array}{l}\text { wl-37256 } \\
\text { wl-37257 }\end{array}$ & $\begin{array}{c}\text { ATTGTTTGCGATGGTGAG } \\
\text { AAAACAATCCAGCAGCAA }\end{array}$ & [23] \\
\hline **Wzy, Serotype O3 & $\begin{array}{l}\text { wl-37258 } \\
\text { wl-37259 }\end{array}$ & $\begin{array}{c}\text { CTCTGTTACTCTCCATAGTGTTC } \\
\text { GATTAGACCACCATAGCCA }\end{array}$ & [23] \\
\hline **Wzy, Serotype O4 & $\begin{array}{l}\text { wl-39105 } \\
\text { wl-39106 }\end{array}$ & $\begin{array}{c}\text { ACTATGGTTTGGCTATACTCCT } \\
\text { ATTCATATCCTGCGTGGC }\end{array}$ & [23] \\
\hline${ }^{* *} W z y$, Serotype O5 & $\begin{array}{l}\text { wl-39873 } \\
\text { wl-39874 }\end{array}$ & $\begin{array}{l}\text { GATGATTTTGTAAGCGGTCT } \\
\text { ACCTACTGGCATAGAGGATAA }\end{array}$ & [23] \\
\hline ** Wzy, Serotype O6 & $\begin{array}{l}\text { wl-40041 } \\
\text { wl-40042 }\end{array}$ & $\begin{array}{l}\text { ATGGTGAAGGGAACGACT } \\
\text { ATCCCCGTGCTATGAGAC }\end{array}$ & [23] \\
\hline ** Wzy, Serotype O7 & $\begin{array}{l}\text { wl-40039 } \\
\text { wl-40040 }\end{array}$ & $\begin{array}{c}\text { CATTTCCAGATTATTACCTTTC } \\
\text { ACACTGGCGATTCTACCC }\end{array}$ & [23] \\
\hline
\end{tabular}

\footnotetext{
${ }^{*}$ Generic primers, ${ }^{* *}$ Cronobacter sakazakii O-antigen serotype specific primers.
} 


\subsection{DNA Sequencing and Data Analysis}

In order to perform DNA sequencing, the amplified PCR products were enzymatically cleaned before cycle sequencing, $3 \mu \mathrm{L}$ of ExoSAP-IT (USB Corporation, Cleveland, OH, USA) was added to $5 \mu \mathrm{L}$ of each amplified PCR product, as described above [13]. The mixture was incubated at $37^{\circ} \mathrm{C}$ for $20 \mathrm{~min}$ followed by $80^{\circ} \mathrm{C}$ for $15 \mathrm{~min}$ on a GeneAmp PCR 9700 thermocycler (Applied Biosystems, Foster City, CA, USA). The purified PCR products were sequenced using AB Big-Dye 3.1 dye chemistry and AB $3500 \mathrm{XL}$ automated DNA sequencers (Applied Biosystems) with sequencing reaction competed for 25 cycles (each cycle is $96^{\circ} \mathrm{C}$ for $30 \mathrm{~s}, 50^{\circ} \mathrm{C}$ for $15 \mathrm{~s}$, and $60^{\circ} \mathrm{C}$ for $4 \mathrm{~min}$ ) and held at $4{ }^{\circ} \mathrm{C}$ in a GeneAmp PCR 9700 Thermocycler (Applied Biosystems). The cycle sequencing reactions contained $2 \mu \mathrm{L}$ of cleaned PCR product, $1 \mu \mathrm{L}$ of BigDye Terminator v3.1 Ready Reaction Mix, $2 \mu \mathrm{L}$ of $5 \times$ Sequencing Buffer, 1.6 pmol of Forward or Reverse sequencing primer, and water in a final volume of $20 \mu \mathrm{L}$. Sequencing reactions were cleaned up with the Performa ${ }^{\circledR}$ DTR Gel Filtration Cartridges following manufacturer's protocol (Edge Bio, Gaithersburg, MD, USA). Sequence accuracy was confirmed by performing two-directional sequencing. Multiple alignments of the generated nucleotide sequences were carried out by using the BioEdit and Geneious programs with manual adjustments.

\subsection{Nucleotide Sequence Accession Numbers}

The generated nucleotide sequences of $16 \mathrm{~S}$ rRNA, rpoB and the seven known C. sakazakii-specific $\mathrm{O}$-antigen genes of the recovered Cronobacter spp. isolates were deposited in the GenBank database under accession numbers KY652858 to KY652894.

\section{Results}

In this surveillance study, a total of 195 enrichments of food samples belonging to nine recognized food categories ( I. baby and medical food; II. dairy products; III. dried food; IV. frozen food; V. pet food; VI. produce; VII. seafood; VIII. spices; and IX. ready-to-eat snacks) were examined initially following conventional microbiologic protocols for the presence of Cronobacter (Table 1). In addition, all 195 food samples were initially tested for the presence of Salmonella, Listeria monocytogenes, and E. coli as part of an ongoing surveillance program for food safety of the agency. There were 14 food samples that tested positive for these three bacterial species known to cause foodborne diseases; Salmonella was detected in half of the 14 food samples tested (data not shown).

Of the various foods examined for Cronobacter, the Cronobacter-specific typical colonies were observed for 51 of the food samples tested (Table 2). The initial biochemical screening for the recovered Cronobacter isolates from typical colonies was achieved by using Biomérieux Vitek 2 System and Cronobacter-specific QPCR (Table 2). All food samples belonging to the baby and medical food and dairy product categories were found to be negative for the presence of Cronobacter. Nevertheless, some of the samples from the rest of the seven food categories were positive for the presence of Cronobacter that included $28.6 \%$ of the dried foods, $20.0 \%$ of the frozen food, $29.1 \%$ of the pet food, $17.8 \%$ of the produce, $9.1 \%$ of the seafood, $44.4 \%$ of the spices, and $23.8 \%$ of the ready-to-eat snacks, food samples investigated (data not shown). Afterward, PCR was performed on these recovered Cronobacter isolates targeting the $16 \mathrm{~S}$ rRNA. rpoB, and the seven Cronobacter sakazakii O-antigen (O1 to $\mathrm{O} 7)$ genes for species identification. Sequence characterization was carried out on the PCR amplified products of seven Cronobacter sakazakii O-antigen (O1 to O7) loci for the identification of Cronobacter sakazakii $\mathrm{O}$ serotypes (Table 4). DNA sequencing of PCR-amplified products of $16 \mathrm{~S}$ rRNA and rpoB genes were also performed for nine isolates (Table 4), which were found to be QPCR positive but PCR-negative for all of the seven O-antigen serotypes primers tested. In all cases, the published primer sets that are listed in Table 3 were tested against the genomic DNA of the specimen with modified PCR conditions at least three times using the HotStarTaq Master Mix kit (QIAGEN), for their sensitivity and robustness by completing PCR amplification. The bi-directional nucleotide sequencing was done on the PCR amplified products for each of the three genes examined. The previously described generic primer sets 
based on the conserved regions of rRNA [21] and rpoB [22] loci known to provide genotypic bacterial identification, resulted in PCR products of approximately $1000 \mathrm{bp}$ and $550 \mathrm{bp}$ in size, for the $16 \mathrm{~S}$ rRNA and rpoB regions amplified, respectively (Figure 1). Furthermore, in this study, the published Cronobacter sakazakii $\mathrm{O}$-antigen (O1 to $\mathrm{O} 7$ ) gene specific primer sets [23] generated the PCR amplified products for six of the seven O-antigen serotypes tested: serotype O1, $364 \mathrm{bp}$; serotype O2,152 bp; serotype O3,704 bp; serotype O4, 890 bp; serotype O6, 424 bp; and serotype O7, 615 bp (Figure 1).

Table 4. Species identification based on $16 \mathrm{~S}$ rRNA and rpoB sequencing for Cronobacter isolates that failed to amplify using O-antigen (1-7) primer sets.

\begin{tabular}{|c|c|c|c|c|c|c|}
\hline $\begin{array}{l}\text { Sample } \\
\text { Number }\end{array}$ & $\begin{array}{l}\text { Description } \\
\text { of Food } \\
\text { Products }\end{array}$ & $\begin{array}{l}\text { Country of } \\
\text { Origin }\end{array}$ & $\begin{array}{l}\text { 16S rRNA Reference * } \\
\text { (Sequence Variation, } \\
\text { \% Similarity) }\end{array}$ & $\begin{array}{c}\text { 16S rRNA } \\
\text { Sequence Type }\end{array}$ & $\begin{array}{c}\text { rpoB Reference }{ }^{* *} \\
\text { (Sequence Variation, } \\
\% \text { Similarity) }\end{array}$ & $\begin{array}{c}\text { rpoB Sequence } \\
\text { Type }\end{array}$ \\
\hline SRL-91 & Pet food & Canada & $\begin{array}{l}\text { GU122174, This report } \\
\text { (Identical, 100\%) } \\
\text { NR_102802, This report } \\
\text { (Identical, 100\%) }\end{array}$ & $\begin{array}{l}\text { C. malonaticus } \\
\text { C. turicensis }\end{array}$ & $\begin{array}{l}\text { CP013940, This report } \\
\text { (8-point-mutation, } 99 \% \text { ) }\end{array}$ & C. malonaticus \\
\hline SRL-12 & Produce & Mexico & $\begin{array}{l}\text { CP004091, This report } \\
\text { (Identical, 100\%) }\end{array}$ & C. sakazakii & $\begin{array}{l}\text { CP013940, This report } \\
\text { (3-point-mutation, 99\%) } \\
\text { JF330141, This report } \\
\text { (3-point-mutation, 99\%) }\end{array}$ & $\begin{array}{l}\text { C. malonaticus } \\
\text { C. sakazakii }\end{array}$ \\
\hline SRL-42 & Produce & Colombia & $\begin{array}{l}\text { KC818225, This report } \\
\text { (2-point-mutation, 99\%) } \\
\text { KU543632, This report } \\
\text { (2-point-mutation, 99\%) }\end{array}$ & $\begin{array}{l}\text { C. malonaticus } \\
\text { C. sakazakii }\end{array}$ & $\begin{array}{l}\text { CP013940, This report } \\
\text { (Identical, 100\%) }\end{array}$ & C. malonaticus \\
\hline SRL-94 & Produce & USA & $\begin{array}{l}\text { KC109002, This report } \\
\text { (2-point-mutation, 99\%) } \\
\text { CP004091, This report } \\
\text { (2-point-mutation, 99\%) } \\
\text { HQ880409, This report } \\
\text { (2-point-mutation, 99\%) }\end{array}$ & $\begin{array}{l}\text { C. malonaticus } \\
\text { C. sakazakii } \\
\text { C. turicensis }\end{array}$ & $\begin{array}{l}\text { CP013940, This report } \\
\text { (Identical, 100\%) }\end{array}$ & C. malonaticus \\
\hline SRL-179 & Spice & India & $\begin{array}{l}\text { KU364464, This report } \\
\text { (Identical, 100\%) }\end{array}$ & C. sakazakii & $\begin{array}{l}\text { JF330150, This report } \\
\text { (Identical, 100\%) }\end{array}$ & C. sakazakii \\
\hline
\end{tabular}

All of the recovered Cronobacter isolates from the 51 food samples were amplified at the 16S rRNA and the rpoB loci (Table 2). Nucleotide sequencing of $16 \mathrm{~S}$ rRNA and the rpoB revealed a considerable inter- as well as intra- specific genetic variation among the recovered Cronobacter isolates characterized, and apparently the $16 \mathrm{~S}$ rRNA regions displayed less polymorphism as compared to the rpoB gene (Table 4).

Of the 51 recovered Cronobacter isolates from the 195 different foods, none of the isolates were found positive for O-antigen 5 serotype; distinct Cronobacter sakazakii $\mathrm{O}$ serotypes were identified among the 51 recovered isolates from various foods (Table 2). However, a significant genetic polymorphism was observed at the rest of the six O-antigen loci sequenced; distinct Cronobacter sakazakii O serotypes were identified among the 51 recovered isolates from various foods (Table 1 ). Three unique 
sequence patterns were noticed among the Cronobacter sakazakii $\mathrm{O}$ serotype 1 isolates; no genetic polymorphisms was observed among the recovered Cronobacter sakazakii $\mathrm{O}$ serotype 2, serotype 3 , and serotype 4 isolates which matched $100 \%$ with respective published sequence available in GenBank (Table 2). The Cronobacter sakazakii O serotype 6 and serotype 7 also displayed unique sequence patterns; some of the sequences matched $100 \%$ with the published sequences and showed minor genetic variation (Table 4).

\section{Discussion}

The primary mission of FDA, being a regulatory agency, is to forbid distribution of hazardous Food, Drug and Cosmetic products, and to keep their supply chain safe. Recently, the remarkable increase in the international production of FDA-regulated commodities (including ingredients and finished products) has made it very challenging to accomplish this mission. This agency uses the presence of human-pathogenic Cronobacter spp. as one of the criteria in implementing regulatory actions and assessing adulteration of foods.

The Cronobacter spp. has been linked primarily to a number of foodborne outbreaks associated with PIF contaminations, and even a lower dose of infection by this pathogen can be life-threatening in neonates $[24,25]$. Since the discovery of Cronobacter, several conventional culture methods have been described for the isolation of Cronobacter spp. [26]. The use of chromogenic selective media with a real-time PCR based confirmatory molecular test was considered to be advantageous for rapid screening and identification of Cronobacter species [27]. In a recent study, it was suggested that incubation at $30^{\circ} \mathrm{C}$ may be suitable for the recovery of some Cronobacter species and minor variations in growth conditions can alter colony morphology and appearance. This may also promote the expression of unique biological characteristics based on phenotypic observations which may be beneficial for differentiating various Cronobacter strains [28].

To date, the ribosomal RNA (rRNA) is considered to be the most conserved region of genomes having several copies and a slower rate of evolution. Therefore, it has been most extensively sequenced to understand the genetic diversity across the prokaryotes and eukaryotes. It has also been most widely used as a phylogenetic marker to understand taxonomic and evolutionary relationships and for the development of molecular diagnostic methods $[3,15,29,30]$. The $16 \mathrm{~S}$ rRNA gene based PCR identification system was reported to be a specific and reliable tool that could correctly identify C. sakazakii isolates from distinct phylogenetic lines [21]. Later, the rpoB gene was described as an effective genetic marker for bacterial identification and phylogeny; a rpoB based PCR systems was developed and evaluated to differentiate the six proposed species within the Cronobacter genus [22,31]. Further, variation in the O-antigen lipopolysaccharide was considered and utilized for serotyping the Gram-negative bacteria. The O-antigen serotyping scheme for C. sakazakii (that includes seven serotypes $\mathrm{O} 1$ to O7) was recently recognized, and the O-antigen gene clusters and specific primers were developed for the identification of $C$. sakazakii $\mathrm{O} 1$ to $\mathrm{O} 7$ strains [23,32]. The sensitivity of PCR assay was described by analyzing the serial dilutions of $C$. sakazakii $\mathrm{O} 1$ to $\mathrm{O} 7$ genomic DNA $(10,1,0.1,0.01,0.001,0.0001$, and $0.00001 \mathrm{ng})$ and using it as a template. The sensitivity of the C. sakazakii $\mathrm{O} 1$ to $\mathrm{O} 7$ isolates in pure culture was also tested by culturing in Luria-Bertani (LB) medium to $\log$ phase followed by 10 -fold serial dilution, and the CFU were counted after overnight incubation at $37^{\circ} \mathrm{C}$. [23]. The seven sets of $\mathrm{O}$-antigen primers were further tested for their sensitivity and specificity by amplifying 136 C. sakazakii $\mathrm{O} 1$ to $\mathrm{O} 7$ isolates, isolates from other Cronobacter species (including two isolates each of C. malonaticus, C. dublinensis and C. turicensis strains, one isolate of C. muytjensii), and 10 isolates of closely related species which also included cross-testing of each primer sets with other $\mathrm{O}$ serotype isolates as well as with the closely related isolates [23]. Results based on the serial dilution (10 to $10^{8} \mathrm{CFU} / \mathrm{mL}$ ) of pure cultures of C. sakazakii O serotypes $\mathrm{O} 1$ to $\mathrm{O} 7$ and using it as templates, revealed positive signals for all seven serotypes at $10^{3} \mathrm{CFU} / \mathrm{mL}$ dilution [23].

Furthermore, multilocus sequence typing (MLST) has been reported suitable for finding genetic polymorphism in microbes with low natural genetic diversity [33-35]. A 7-loci based Cronobacter-specific 
MLST was developed [36], and the MLST was subsequently used to understand genetic diversity of recovered C. sakazakii isolates from PIF, ingredients of PIF, and their production premises [37-41]. More recently, MLST was employed in the genetic characterization of Cronobacter sakazakii recovered from the environmental surveillance samples during a sporadic case investigation of foodborne illness [17].

In recent years, a number of surveillance studies have been carried out for the identification of Cronobacter spp. from foods, using conventional microbiological and molecular techniques worldwide. These surveillance studies include research on raw dried pasta from the German market [42], wheat flour from China [43], dehydrated rice powder from the Chinese Supermarket [44], dried food from Japan [45], retails foods from Brazil and Czech Republic [11,46], ready-to-eat foods other than infant formula from Ireland and Switzerland [47], ready-to-eat foods from China [48], herbs and spices from Jordon [49], medicinal plants, herbs, and spices from India [50], spices and herbs from Poland [51], infant formula production factory premises and powdered infant formula from China [52]. The molecular tools were also successfully used in the reevaluation of a suspected Cronobacter sakazakii outbreak in Mexico [53].

\section{Conclusions}

The $16 \mathrm{~S}$ rRNA, rpoB and the seven Cronobacter sakazakii-specific O-antigen primer sets can be used for rapid detection and differentiation of Cronobacter spp. isolates recovered from the surveillance food samples. DNA sequencing of O-antigen 1-7 serotyping is an ideal tool for the genetic typing of C. sakazakii recovered isolates from foods. These unique molecular diagnostic tools can help the FDA accomplish its important mandate of the food safety program, and conduct epidemiologic surveillance and investigations of public health importance.

Acknowledgments: This study was supported in part by funding from the Southeast Regional Laboratory of FDA. The funders had no role in the study design, data collection and analysis, decision to publish, or preparation of the manuscript. We also thank Nicky Sulaiman of CDC for her help for the completion of this work.

Author Contributions: N.M. and I.M.S. conceived and designed the experiments; N.M. performed the experiments; P.B. and I.M.S. analyzed the data; S.S. and K.K. contributed reagents/materials/analysis tools; N.M., P.B., and I.M.S. wrote the paper.

Conflicts of Interest: The authors declare no conflict of interest.

Disclaimer: The findings and conclusions made in this manuscript are those of the authors and do not necessarily represent the views or official position of the U.S. Food and Drug Administration (FDA). The names of vendors or manufacturers are provided as examples of available product sources; inclusion does not imply endorsement of the vendors; manufacturers; or products by the FDA or the U.S. Department of Health and Human Services.

\section{References}

1. Centers for Disease Control Prevention. Enterobacter sakazakii infections associated with the use of powdered infant formula-Tennessee, 2001. Morb. Mortal. Wkly. Rep. 2002, 51, 297-300.

2. Lai, K.K. Enterobacter sakazakii Infections among Neonates, Infants, Children, and Adults. Medicine 2001, 80, 113-122. [CrossRef] [PubMed]

3. Osaili, T.; Forsythe, S. Desiccation resistance and persistence of Cronobacter species in infant formula. Int. J. Food Microbiol. 2009, 136, 214-220. [CrossRef] [PubMed]

4. Iversen, C.; Mullane, N.; McCardell, B.; Tall, B.D.; Lehner, A.; Fanning, S.; Stephan, R.; Joosten, H. Cronobacter gen. nov., a new genus to accommodate the biogroups of Enterobacter sakazakii, and proposal of Cronobacter sakazakii gen. nov., comb. nov., Cronobacter malonaticus sp. nov., Cronobacter turicensis sp. nov., Cronobacter muytjensii sp. nov., Cronobacter dublinensis sp. nov., Cronobacter genomospecies 1, and of three subspecies, Cronobacter dublinensis subsp. dublinensis subsp. nov., Cronobacter dublinensis subsp. lausannensis subsp. nov. and Cronobacter dublinensis subsp. lactaridi subsp. nov. Int. J. Syst. Evol. Microbiol. 2008, 58, 1442-1447. [PubMed] 
5. Jarvis, K.G.; Yan, Q.Q.; Grim, C.J.; Power, K.A.; Franco, A.A.; Hu, L.; Gopinath, G.; Sathyamoorthy, V.; Kotewicz, M.L.; Kothary, M.H. Identification and Characterization of Five New Molecular Serogroups of Cronobacter spp. Foodborne Pathog. Dis. 2013, 10, 343-352. [CrossRef] [PubMed]

6. Joseph, S.; Cetinkaya, E.; Drahovska, H.; Levican, A.; Figueras, M.J.; Forsythe, S.J. Cronobacter condimenti sp. nov., isolated from spiced meat, and Cronobacter universalis sp. nov., a species designation for Cronobacter sp. genomospecies 1, recovered from a leg infection, water and food ingredients. Int. J. Syst. Evol. Microbiol. 2011, 62, 1277-1283. [CrossRef] [PubMed]

7. Joseph, S.; Sonbol, H.; Hariri, S.; Desai, P.; McClelland, M.; Forsythe, S.J. Diversity of the Cronobacter Genus as Revealed by Multilocus Sequence Typing. J. Clin. Microbiol. 2012, 50, 3031-3039. [CrossRef] [PubMed]

8. Kandhai, M.C.; Reij, M.W.; Grognou, C.; van Schothorst, M.; Gorris, L.G.; Zwietering, M.H. Effects of preculturing conditions on lag time and specific growth rate of Enterobacter sakazakii in reconstituted powdered infant formula. Appl. Environ. Microbiol. 2006, 72, 2721-2729. [CrossRef] [PubMed]

9. Strydom, A.; Cameron, M.; Witthuhn, R.C. Phylogenetic Analysis of Cronobacter Isolates Based on the rpoA and 16S rRNA Genes. Curr. Microbiol. 2012, 64, 251-258. [CrossRef] [PubMed]

10. Jason, J. The Roles of Epidemiologists, Laboratorians, and Public Health Agencies in Preventing Invasive Cronobacter Infection. Front. Pediatr. 2015, 3, 110. [CrossRef] [PubMed]

11. Hochel, I.; R̛̊žičková, H.; Krásný, L.; Demnerová, K. Occurrence of Cronobacter spp. in retail foods. J. Appl. Microbiol. 2012, 112, 1257-1265. [CrossRef] [PubMed]

12. Carter, L.; Lindsey, L.A.; Grim, C.J.; Sathyamoorthy, V.; Jarvis, K.G.; Gopinath, G.; Lee, C.; Sadowski, J.A.; Trach, L.; Pava-Ripoll, M. Multiplex PCR Assay Targeting a Diguanylate Cyclase-Encoding Gene, cgcA, to Differentiate Species within the Genus Cronobacter. Appl. Environ. Microbiol. 2012, 79, 734-737. [CrossRef] [PubMed]

13. Sulaiman, I.; Jacobs, E.; Simpson, S.; Kerdahi, K. Genetic Characterization of Fungi Isolated from the Environmental Swabs collected from a Compounding Center Known to Cause Multistate Meningitis Outbreak in United States Using ITS Sequencing. Pathogens 2014, 3, 732-742. [CrossRef] [PubMed]

14. Sulaiman, I.; Jacobs, E.; Simpson, S.; Kerdahi, K. Molecular Identification of Isolated Fungi from Unopened Containers of Greek Yogurt by DNA Sequencing of Internal Transcribed Spacer Region. Pathogens 2014, 3, 499-509. [CrossRef] [PubMed]

15. Sulaiman, I.M.; Ortega, Y.; Simpson, S.; Kerdahi, K. Genetic characterization of human-pathogenic Cyclospora cayetanensis parasites from three endemic regions at the $18 \mathrm{~S}$ ribosomal RNA locus. Infect. Genet. Evol. 2014, 22, 229-234. [CrossRef] [PubMed]

16. Yan, Q.; Fanning, S.A. Strategies for the Identification and Tracking of Cronobacter Species: An Opportunistic Pathogen of Concern to Neonatal Health. Front. Pediatr. 2015, 3, 38. [CrossRef] [PubMed]

17. Sulaiman, I.M.; Jacobs, E.; Segars, K.; Simpson, S.; Kerdahi, K. Genetic Characterization of Cronobacter sakazakii Recovered from the Environmental Surveillance Samples During a Sporadic Case Investigation of Foodborne Illness. Curr. Microbiol. 2016, 73, 273-279. [CrossRef] [PubMed]

18. U.S. Food and Drug Administration Bacteriological Analytical Manual (8th Edition). Available online: https://www.fda.gov/food/foodscienceresearch/laboratorymethods/ucm114664.htm (accessed on 24 February 2017).

19. Al-Holy, M.A.; Shin, J.H.; Osaili, T.M.; Rasco, B.A. Evaluation of a New Enrichment Broth for Detection of Cronobacter spp. in Powdered Infant Formula. J. Food Prot. 2011, 74, 387-393. [CrossRef] [PubMed]

20. Pincus, D.H. Microbial Identification Using the BioMerieux VITEK ${ }^{\circledR} 2$ System. In Encyclopedia of Rapid Microbiological Methods; Parenteral Drug Association: Bethesda, MD, USA, 2006.

21. Lehner, A.; Tasara, T.; Stephan, R. 16S rRNA gene based analysis of Enterobacter sakazakii strains from different sources and development of a PCR assay for identification. BMC Microbiol. 2004, 4, 43. [CrossRef] [PubMed]

22. Mollet, C.; Drancourt, M.; Raoult, D. rpoB sequence analysis as a novel basis for bacterial identification. Mol. Microbiol. 1997, 26, 1005-1011. [CrossRef] [PubMed]

23. Sun, Y.; Wang, M.; Wang, Q.; Cao, B.; He, X.; Li, K.; Feng, L.; Wang, L. Genetic Analysis of the Cronobacter sakazakii $\mathrm{O} 4$ to O7 O-Antigen Gene Clusters and Development of a PCR Assay for Identification of All C. sakazakii O Serotypes. Appl. Environ. Microbiol. 2012, 78, 3966-3974. [CrossRef] [PubMed]

24. Bowen, A.B.; Braden, C.R. Invasive Enterobacter sakazakii Disease in Infants. Emerg. Infect. Dis. 2006, 12, 1185-1189. [CrossRef] [PubMed] 
25. Iversen, C.; Forsythe, S. Risk profile of Enterobacter sakazakii, an emergent pathogen associated with infant milk formula. Trends Food Sci.Technol. 2003, 14, 443-454. [CrossRef]

26. Muytjens, H.L.; Roelofs-Willemse, H.; Jaspar, G.H. Quality of powdered substitutes for breast milk with regard to members of the family Enterobacteriaceae. J. Clin. Microbiol. 1988, 26, 743-746. [PubMed]

27. Chen, Y.I.; Song, K.-Y.; Brown, E.W.; Lampel, K.A. Development of an Improved Protocol for the Isolation and Detection of Enterobacter sakazakii (Cronobacter) from Powdered Infant Formula. J. Food Prot. 2010, 73, 1016-1022. [CrossRef] [PubMed]

28. Segars, K.; Simpson, S.; Kerdahi, K.; Sulaiman, I.M. Evaluation of Cronobacter Growth and Phenotypic Variation Under Modified Culture Conditions. Curr. Microbiol. 2015, 72, 190-197. [CrossRef] [PubMed]

29. Sulaiman, I.M.; Fayer, R.; Lal, A.A.; Trout, J.M.; Schaefer, F.W.; Xiao, L. Molecular Characterization of Microsporidia Indicates that Wild Mammals Harbor Host-Adapted Enterocytozoon spp. as well as Human-Pathogenic Enterocytozoon bieneusi. Appl. Environ. Microbiol. 2003, 69, 4495-4501. [CrossRef] [PubMed]

30. Sulaiman, I.M.; Lal, A.A.; Xiao, L. A Population Genetic Study of the Cryptosporidium parvum Human Genotype Parasites. J. Eukaryot. Microbiol. 2001, 48, 24S-27S. [CrossRef]

31. Stoop, B.; Lehner, A.; Iversen, C.; Fanning, S.; Stephan, R. Development and evaluation of rpoB based PCR systems to differentiate the six proposed species within the genus Cronobacter. Int. J. Food Microbiol. 2009, 136, 165-168. [CrossRef] [PubMed]

32. Sun, Y.; Wang, M.; Liu, H.; Wang, J.; He, X.; Zeng, J.; Guo, X.; Li, K.; Cao, B.; Wang, L. Development of an O-Antigen Serotyping Scheme for Cronobacter sakazakii. Appl. Environ. Microbiol. 2011, 77, 2209-2214. [CrossRef] [PubMed]

33. Sulaiman, I.M.; Anderson, M.; Khristova, M.; Tang, K.; Sulaiman, N.; Phifer, E.; Simpson, S.; Kerdahi, K. Development of a PCR-Restriction Fragment Length Polymorphism Protocol for Rapid Detection and Differentiation of Four Cockroach Vectors (Group I “Dirty 22" Species) Responsible for Food Contamination and Spreading of Foodborne Pathogens: Public Health Importance. J. Food Prot. 2011, 74, 1883-1890. [PubMed]

34. Sulaiman, I.M.; Anderson, M.; Oi, D.H.; Simpson, S.; Kerdahi, K. Multilocus Genetic Characterization of Two Ant Vectors (Group II “Dirty 22” Species) Known To Contaminate Food and Food Products and Spread Foodborne Pathogens. J. Food Prot. 2012, 75, 1447-1452. [CrossRef] [PubMed]

35. Sulaiman, I.M.; Torres, P.; Simpson, S.; Kerdahi, K.; Ortega, Y. Sequence Characterization of Heat Shock Protein Gene of Cyclospora cayetanensis Isolates from Nepal, Mexico, and Peru. J. Parasitol. 2013, 99, 379-382. [CrossRef] [PubMed]

36. Baldwin, A.; Loughlin, M.; Caubilla-Barron, J.; Kucerova, E.; Manning, G.; Dowson, C.; Forsythe, S. Multilocus sequence typing of Cronobacter sakazakii and Cronobacter malonaticus reveals stable clonal structures with clinical significance which do not correlate with biotypes. BMC Microbiol. 2009, 9, 223. [CrossRef] [PubMed]

37. Cetinkaya, E.; Joseph, S.; Ayhan, K.; Forsythe, S.J. Comparison of methods for the microbiological identification and profiling of Cronobacter species from ingredients used in the preparation of infant formula. Mol. Cell. Probes 2013, 27, 60-64. [CrossRef] [PubMed]

38. Gičová, A.; Oriešková, M.; Oslanecová, L.; Drahovská, H.; Kaclíková, E. Identification and characterization of Cronobacter strains isolated from powdered infant foods. Lett. Appl. Microbiol. 2013, 58, 242-247. [CrossRef] [PubMed]

39. Killer, J.; Skřivanová, E.; Hochel, I.; Marounek, M. Multilocus Sequence Typing of Cronobacter Strains Isolated from Retail Foods and Environmental Samples. Foodborne Pathog. Dis. 2015, 12, 514-521. [CrossRef] [PubMed]

40. Müller, A.; Stephan, R.; Fricker-Feer, C.; Lehner, A. Genetic Diversity of Cronobacter sakazakii Isolates Collected from a Swiss Infant Formula Production Facility. J. Food Prot. 2013, 76, 883-887. [CrossRef] [PubMed]

41. Sonbol, H.; Joseph, S.; McAuley, C.M.; Craven, H.M.; Forsythe, S.J. Multilocus sequence typing of Cronobacter spp. from powdered infant formula and milk powder production factories. Int. Dairy J. 2013, 30, 1-7. [CrossRef]

42. Akineden, Ö.; Murata, K.J.; Gross, M.; Usleber, E. Microbiological Quality of Raw Dried Pasta from the German Market, with Special Emphasis on Cronobacter Species. J. Food Sci. 2015, 80, M2860-M2867. [CrossRef] [PubMed] 
43. Lou, X.; Si, G.; Yu, H.; Qi, J.; Liu, T.; Fang, Z. Possible reservoir and routes of transmission of Cronobacter (Enterobacter sakazakii) via wheat flour. Food Control 2014, 43, 258-262. [CrossRef]

44. Huang, Y.; Pang, Y.; Wang, H.; Tang, Z.; Zhou, Y.; Zhang, W.; Li, X.; Tan, D.; Li, J.; Lin, Y. Occurrence and Characterization of Cronobacter spp. in Dehydrated Rice Powder from Chinese Supermarket. PLoS ONE 2015, 10, e0131053. [CrossRef] [PubMed]

45. Ogihara, H.; Kiribe, N.; Fukuda, N.; Furukawa, S.; Morinaga, Y.; Igimi, S. Cronobacter spp. in Commercially Available Dried Food in Japan. Biocontrol Sci. 2014, 19, 209-213. [CrossRef] [PubMed]

46. Brandão, M.L.L.; Umeda, N.S.; Jackson, E.; Forsythe, S.J.; de Filippis, I. Isolation, molecular and phenotypic characterization, and antibiotic susceptibility of Cronobacter spp. from Brazilian retail foods. Food Microbiol. 2017, 63, 129-138. [CrossRef] [PubMed]

47. Baumgartner, A.; Grand, M.; Liniger, M.; Iversen, C. Detection and frequency of Cronobacter spp. (Enterobacter sakazakii) in different categories of ready-to-eat foods other than infant formula. Int. J. Food Microbiol. 2009, 136, 189-192. [CrossRef] [PubMed]

48. Xu, X.; Li, C.; Wu, Q.; Zhang, J.; Huang, J.; Yang, G. Prevalence, molecular characterization, and antibiotic susceptibility of Cronobacter spp. in Chinese ready-to-eat foods. Int. J. Food Microbiol. 2015, 204, 17-23. [CrossRef] [PubMed]

49. Jaradat, Z.W.; Ababneh, Q.O.; Saadoun, I.M.; Samara, N.A.; Rashdan, A.M. Isolation of Cronobacter spp. (formerly Enterobacter sakazakii) from infant food, herbs and environmental samples and the subsequent identification and confirmation of the isolates using biochemical, chromogenic assays, PCR and 16S rRNA sequencing. BMC Microbiol. 2009, 9, 225. [CrossRef] [PubMed]

50. Singh, N.; Goel, G.; Raghav, M. Prevalence and Characterization of Cronobacter spp. from Various Foods, Medicinal Plants, and Environmental Samples. Curr. Microbiol. 2015, 71, 31-38. [CrossRef] [PubMed]

51. Garbowska, M.; Berthold-Pluta, A.; Stasiak-Różańska, L. Microbiological quality of selected spices and herbs including the presence of Cronobacter spp. Food Microbiol. 2015, 49, 1-5. [CrossRef] [PubMed]

52. Fei, P.; Man, C.; Lou, B.; Forsythe, S.J.; Chai, Y.; Li, R.; Niu, J.; Jiang, Y. Genotyping and Source Tracking of Cronobacter sakazakii and C. malonaticus Isolates from Powdered Infant Formula and an Infant Formula Production Factory in China. Appl. Environ. Microbiol. 2015, 81, 5430-5439. [CrossRef] [PubMed]

53. Jackson, E.E.; Flores, J.P.; Fernández-Escartín, E.; Forsythe, S.J. Reevaluation of a Suspected Cronobacter sakazakii Outbreak in Mexico. J. Food Prot. 2015, 78, 1191-1196. [CrossRef] [PubMed] 